\title{
Querschnittrehabilitation fördert den Weg zurück in ein aktives Leben
}

\section{Xavier Jordan}

Dr. med., Clinique romande de réadaptation, Sion

\section{Hochspezialisierte Rehabilitation verbessert nicht nur die Selbständigkeit und die Lebens-} qualität der querschnittgelähmten Patienten erheblich. Auch der ökonomische Nutzen ist hoch, wenn Patienten keine oder weniger Pflege- und Hilfsleistungen benötigen, weniger Folgekomplikationen entwickeln und durch ihre Berufstätigkeit höhere Berentungen entfallen.

In der Schweiz erleiden jedes Jahr mehr als 250 Menschen eine Querschnittlähmung: Mehrheitlich sind es junge Menschen mit Rückenmarkverletzungen, nach Verkehr- oder Arbeitsunfällen oder aber durch Sportunfälle vom Gleitschirmfliegen, Baden, Ski- und Velofahren oder Reiten. Die zweite grosse Patientengruppe ist deutlich älter und erleidet eine Querschnittlähmung aufgrund einer Krankheit wie eines Bandscheibenvorfalles, eines Tumors oder einer fortschreitenden Erkrankung wie der Multiplen Sklerose.

\section{Querschnittgelähmte Patienten sind mit vielen Herausforderungen konfrontiert}

Eine Querschnittlähmung stellt eine enorme Herausforderung an die physische und psychische Anpassungsfähigkeit eines jeden Betroffenen und seines Umfeldes. Die durch die Verletzung des Rückenmarks verursachten Verluste von Bewegungsfähigkeit und Sensibilität stellen für die betroffenen Personen eine deutliche Einschränkung in ihrer Alltagsbewältigung dar und bringen das ganze bisherige Leben aus dem Lot.

Darüber hinaus verursachen Querschnittlähmungen eine ganze Reihe an erheblichen, aber weniger sichtbaren Komplikationen, welche jedoch lebensbedrohlich werden können. Neben Ausfällen oder Einschränkungen der Blasen-, Darm- und Sexualfunktionen können aufgrund der fehlenden Mobilität und der langen Liege- oder Sitzzeiten Druckgeschwüre (Dekubitus) entstehen, welche die Patienten zu mehrwöchigen Hospitalisationen zwingen können. Häufig entwickelt sich auch schon sehr früh eine erhöhte Muskelspannung, die unerwünschte und störende Haltungen erzwingt (Spastizität, Kontrakturen) und Schmerzen, was nur durch Medikation in einem erträglichen Rahmen gehalten werden kann. Ebenfalls häufige Folgen sind sozialer Rückzug und depressive Störungen.

Noch weitere, weniger bekannte Komplikationen treten bei einer Verletzung des Nervensystems öfter auf: So kann durch die Entgleisung im autonomen Nervensystem der Blutdruck sehr tief sein (orthostatische Hypotonie) oder hoch hinaus schiessen (autonome Dysreflexie), die Knochen werden aufgrund der fehlenden Belastung dünn (Osteoporose) und brüchig (Frakturen), und die wenig bewegten Gelenke können verknöchern (heterotrope Ossifikationen). Je nach Lähmungshöhe können schon sehr früh Herz- und Lungen-Funktionsstörungen auftreten. Durch die fehlenden Muskelbewegungen entstehen tiefe Beinvenenthrombosen, aus denen sich eine tödliche Lungenembolie entwickeln kann. All diese Probleme schränken die Lebensqualität und bis heute auch die Lebenserwartung der Betroffenen ein. Sie verringern darüber hinaus Autonomie und Mobilität und stören die Teilhabe am sozialen und beruflichen Leben erheblich [1].

Werden Patienten rasch in ein Spezialzentrum verlegt, sterben sie seltener und erleiden weniger Komplikationen.

\section{Der medizinische Fortschritt bringt heute deutlich bessere Behandlungsergebnisse}

Vor dem Hintergrund dieses komplexen Bildes ist die Betreuung querschnittgelähmter Menschen vom Auftritt der Lähmung bis zum Lebensende anspruchsvoll. Die Fortschritte seit Beginn der systematischen medizinischen Betreuung im Zweiten Weltkrieg sind beeindruckend. Die Lebenserwartung Querschnittgelähmter hat sich zwischen 1950 und 1980 deutlich verlängert 
[2], unter anderem durch die Entwicklung der Blasenkatheter und der damit verbundenen Möglichkeit einer regelmässigen und gesicherten Blasenentleerung, welche die frühe Entwicklung eines oftmals tödlichen Nierenversagens verhindern. Neueste Daten zeigen ein Anhalten dieses Trends, wenn auch in kleinerem Ausmass. Dass dabei der spezialisierten Betreuung in Querschnittzentren eine wichtige Rolle zufällt, zeigen bereits Arbeiten aus den 1990er Jahren. Eine rasche Verlegung (i.e. <30 Tagen) von Menschen mit traumatischer Rückenmarklähmung in ein Spezialzentrum hat zur Folge, dass die Patienten seltener sterben, weniger und leichtere Komplikationen erleiden und dennoch kürzere stationäre Behandlungen in Anspruch nehmen [3, 4].

Die Betreuung im Spezialzentrum ist nämlich genau auf die Bedürfnisse dieser Patienten zugeschnitten. In der ersten Phase müssen die Frühkomplikationen nach Auftreten der Querschnittlähmung erkannt und abgefangen werden. Dies bedarf einer spezifischen Ausbildung des Fachpersonals. Querschnittgelähmte Menschen müssen in einer zweiten Phase lernen, mit ihrem veränderten Körper umzugehen und die hierfür nötigen Massnahmen selbständig durchzuführen. Dabei müssen sie viel Arbeit und Geduld in die möglichst grosse Verbesserung ihrer Lähmung investieren, was nur in der genau auf ihre Bedürfnisse zugeschnittenen Struktur eines Spezialzentrums erfolgen kann. Darüber hinaus müssen Spätkomplikationen möglichst verhindert werden, indem jeder Querschnittgelähmte lernt, deren erste Anzeichen zu erkennen und ihnen mit dem richtigen Verhalten zu begegnen. Dafür werden sie vom Fachpersonal begleitet und geschult.

\section{Die spezialisierte Rehabilitation ermöglicht eine schnellere Rückkehr in das soziale Umfeld und reduziert den Pflegebedarf.}

Die vielfältigen Auswirkungen einer Rückenmarkverletzung auf die Gesundheit machen die medizinische Betreuung besonders komplex und anspruchsvoll. Die WHO hat die Querschnittversorgung als hochspezialisiert anerkannt und hält ihre Fortschritte, vor allem in Bezug auf das Überleben der Patienten, ihre Selbständigkeit, ihre Lebensqualität und das Zurückfinden ins Berufsleben fest [5]. So erlaubt die Querschnittrehabilitation in spezialisierten Zentren den Betroffenen häufiger eine Rückkehr in ihr soziales Umfeld bzw. zu ihren Familien, so dass weniger Platzierungen in Heimen und anderen Pflegeinstitutionen erfolgen müssen [6]. All diese Fortschritte konnten vor allem durch die lebenslange Nachbetreuung der Patienten in spezialisierten Sprechstunden von Querschnittspezialisten erreicht werden.
Die beschriebenen sekundären Komplikationen sind in den ersten Jahren nach Eintritt der Querschnittlähmung sehr häufig und können tödlich enden, wenn sie nicht rasch erkannt und optimal behandelt werden. Aber auch nach dieser ersten Phase können sich allmählich Spätkomplikationen entwickeln, deren Verlauf bei korrekter Nachsorge, verlangsamt werden kann. Hierzu müssen speziell ausgebildete Fachpersonen die Patienten regelmässig und systematisch kontrollieren, spezifische Screening-Untersuchungen durchführen und im Gespräch mit Patienten und ihrem Umfeld die nötigen Anpassungen empfehlen, besprechen und einleiten. Dies verleiht der lebenslangen Nachsorge in einem gut koordinierten, interdisziplinären Setting in spezialisierten Institutionen grosse Bedeutung [7]. Nur so können die Autonomie,

\section{Lebenslange Nachsorge gibt querschnitt- gelähmten Menschen die Möglichkeit, ihren Platz in der Gesellschaft einzunehmen.}

die Lebensqualität, die Inklusion und die Teilhabe unserer querschnittgelähmten Mitmenschen unter bestmöglicher Bewahrung ihres Gesundheitszustandes gewährleistet werden, so dass sie ihren Platz in der Gesellschaft einnehmen und halten können.

Für querschnittgelähmte Patienten wird in den nächsten Jahren entscheidend sein, welche Weichen die Gesundheitspolitik stellt. Änderungen des Tarifsystems, die zu stark auf kurzfristige Effekte zielen, könnten die ganzheitliche Versorgung, wie sie von der WHO und der ISCoS [8] empfohlen wird, gefährden. Würden die durch eine Querschnittlähmung verursachten hohen Kosten [9] weiterhin übernommen? Das heute hohe Qualitätsniveau in der Akut- und langfristigen Behandlung Querschnittsgelähmter resultiert aus den Anstrengungen der letzten 50 Jahre seit 1963 das erste Querschnittzentrum in Genf eröffnet wurde und nähert sich den Zielen der Konvention über die Rechte von Menschen mit Behinderungen [10], die die Schweiz 2014 ratifiziert hat. Eine Eingrenzung des Zuganges zu spezialisierten medizinischen Leistungen wäre eine ethische und moralische Niederlage. Der wachsende Druck auf Institutionen und Leistungserbringer könnte die Anstrengungen zur Verbesserung der sozialen Integration und Lebensqualität der Patienten und ihrer Familien ausbremsen. Dies hätte mittel- und langfristig auch auf volkswirtschaftlicher Ebene negative Auswirkungen, da mehr gesundheitliche Komplikationen, Arbeitsausfälle und Invaliditätsrenten höhere Kosten verursachen würden. 